\title{
Postoperative Pharmacologic Anticoagulation following Temporal Lobe Resection of a Gliosarcoma in a Hypercoagulable Patient
}

\author{
Alec Kellish, BS \\ Cooper Medical School of Rowan University, kellisha5@rowan.edu \\ Siyuan Yu, BS \\ Cooper Medical School of Rowan University, siyuan1005@gmail.com \\ Mark Heslin, BS \\ Cooper Medical School of Rowan University, heslinm6@rowan.edu \\ Brian Gable, MD \\ Cooper Medical School of Rowan University, gable@rowan.edu \\ Gabrielle Hassinger, MD \\ Cooper University Hospital, hassinger-gabrielle1@cooperhealth.edu \\ Cooper Rowan Medical Journal: https://rdw.rowan.edu/crjcsm
}

Would you like to be a reviewer? Please fill in this short form to express your interest.

\section{Recommended Citation}

Kellish, BS, Alec; Yu, BS, Siyuan; Heslin, BS, Mark; Gable, MD, Brian; and Hassinger, MD, Gabrielle (2021)

"Postoperative Pharmacologic Anticoagulation following Temporal Lobe Resection of a Gliosarcoma in a Hypercoagulable Patient," Cooper Rowan Medical Journal: Vol. 3 : Iss. 1 , Article 2.

DOI: 10.31986issn.2578.3343_vol2iss1.9

Available at: https://rdw.rowan.edu/crjcsm/vol3/iss1/2

\section{(c) (7)}

This work is licensed under a Creative Commons Attribution 4.0 License.

This Case Reports and Case Series is brought to you for free and open access by the Rowan University Journals at Rowan Digital Works. It has been accepted for inclusion in Cooper Rowan Medical Journal by an authorized editor of Rowan Digital Works. For more information, please contact brush@rowan.edu. 
Postoperative Pharmacologic Anticoagulation following Temporal Lobe Resection of a Gliosarcoma in a Hypercoagulable Patient 


\title{
Postoperative Pharmacologic Anticoagulation following Temporal Lobe Resection of a Gliosarcoma in a Hypercoagulable Patient
}

\author{
Alec Kellish, BS ${ }^{1 *}$, Siyuan Yu, BS ${ }^{1}$, Mark Heslin, BS ${ }^{1}$, Gabrielle Hassinger, MD $^{2}$ \& \\ Brian Gable, $\mathrm{MD}^{3}$ \\ ${ }^{1}$ Student Doctor, Cooper Medical School of Rowan University, Camden, 08103, NJ \\ ${ }^{2}$ Resident Physician, Internal Medicine, Cooper University Hospital, Camden, 08103, New Jersey \\ ${ }^{3}$ Head of Medical Education, Cooper Medical School of Rowan University, Camden, 08103, NJ \\ *Corresponding author: kellisha5@rowan.edu (Alec Kellish, BS)
}

\begin{abstract}
Gliosarcomas are a rare subtype of glioblastomas associated with high rates of malignancy-associated venous thromboembolism (VTE). VTE risk is further increased in hypercoagulable patients upon discontinuing pharmacologic anticoagulation for surgery. We present a 60-year old obese male with history of hypercoagulability on apixaban who developed extensive thrombosis following resection of a gliosarcoma. Prior to temporal lobe resection, apixaban was discontinued and an IVC filter placed. On postoperative day 4, imaging revealed thrombosis above the IVC filter extending to the bilateral common, internal and external iliac, and femoral veins, requiring immediate anticoagulation and suction thrombectomy. Clinicians must balance the risk of VTE and intracerebral hemorrhage following neurosurgical procedures. While withholding pharmacologic VTE is standard, hypercoagulable patients may benefit from earlier institution of pharmacologic prophylaxis postoperatively. Patients with multiple risk factors including malignancies with high rates VTE, like gliosarcomas with medical and hematological conditions that predispose to clotting, including idiopathic erythrocytosis and history of VTE may benefit from earlier pharmacologic prophylaxis.
\end{abstract}




\section{INTRODUCTION}

Glioblastomas are the most common primary malignant brain tumors in adults with a median age of diagnosis around 55-60 years old. A rare histopathologic subtype of glioblastomas are gliosarcomas, an isocitrate dehydrogenase wild type glioblastoma, comprising approximately 2-3\% of all WHO Grade IV primary glioblastomas. ${ }^{1,2}$

All glioblastomas are derived from astrocytes, the most common type of glial cell. Glioblastomas are a heterogenous group of malignant tumors without a common genetic signature and varied histopathology, location, and genetic mutations. ${ }^{3}$ The gliosarcoma variation of glioblastomas can occur sporadically as a primary malignancy, or be a secondary malignancy following radiation to the brain. ${ }^{4}$ Gliosarcomas display biphasic histopathology, containing gliomatous differentiation, present in all glioblastomas, as well as mesenchymal components, classically associated with sarcomas, and high grade malignant cells with areas of necrosis, mitosis and atypia. ${ }^{5}$ These mesenchymal components resemble histopathology associated with liposarcoma, chondrosarcoma, and osteosarcomas. ${ }^{6,7}$ Clinically, gliosarcomas have been traditionally viewed similarly to other grade IV glioblastomas, sharing similar rates of survival, response to treatments, and rates of recurrence. ${ }^{8-10}$

A challenging aspect of management shared by glioblastomas and gliosarcomas is the risk of venous thromboembolism (VTE), especially after neurosurgical intervention. Malignancies dramatically increase the risk of VTE by more than five-fold, with $30 \%$ of all VTE occurring in the setting of malignancy. ${ }^{11,12}$ The rate of VTE in high grade gliomas is $20-30 \%$, and as high as $25-39 \%$ in patients with high grade glioblastoma. ${ }^{11,13}$ Pharmacologic prophylaxis and intervention for postoperative VTE is complicated following intracranial surgery. The risk of VTE must be weighed against the risk anticoagulation leading to intracerebral hemorrhage (ICH), which occurs in nearly $2 \%$ of patients following intracranial tumor resection. ${ }^{14}$

We present a case of a patient with a history of hypercoagulability and deep vein thrombosis (DVT) in the setting of idiopathic erythrocytosis on chronic anticoagulation who developed extensive VTE after resection of a grade IV gliosarcoma.

\section{Case Report}

A 60-year-old obese male with a history of hypertension, diabetes mellitus, idiopathic erythrocytosis requiring intermittent phlebotomy and DVT in the setting of erythrocytosis, currently on apixaban presented to the emergency department with a two-week history of unilateral, pulsating, temporal headaches, which progressed to 10/10 pain on the day of admission. Associated symptoms include nausea, blurry vision, and difficulty with ambulation. Of note, the idiopathic erythrocytosis work up eight years ago for genetic and paraneoplastic etiologies was inconclusive; negative for JAK2, MPL, and calreticulin 
mutations and no renal or liver masses were present. Three years ago, the patient developed a lower extremity DVT secondary to erythrocytosis, underwent a thrombectomy and has been on apixaban for future DVT prophylaxis.

On physical exam, no focal neurological deficits were appreciated, however the fundoscopic exam revealed bilateral papilledema. A non-contrast CT head showed a $3.8 \mathrm{~cm}$ right temporal mass with vasogenic edema and $0.4 \mathrm{~cm}$ right to left midline shift. A follow-up magnetic resonance imaging demonstrated an enhancing cystic and solid mass, and neurosurgical resection as deemed necessary (Figure 1). A right temporal craniectomy with resection of the temporal lobe mass was performed. Histopathological analysis of the specimen demonstrated a biphasic malignant tumor with a mixture of gliomatous and mesenchymal components. The glial component showed gemistocytic areas, epithelioid morphology, and primitive cellular areas with a high nuclear to cytoplasmic ratio. The mesenchymal component represented spindle cell sarcoma that was reticulin rich on histochemical staining, which was consistent with WHO Grade IV gliosarcoma. Prior to his operation, apixaban was held and a retrievable inferior vena cava (IVC) filter was placed for VTE prophylaxis. Following the resection, the patient had no neurological deficits, and remained on mechanical VTE prophylaxis. The patient was not restarted on pharmaceutical anticoagulation due to the potential risk of ICH causing a catastrophic bleed. Additionally, he received a unit of platelets on postoperative day three due to thrombocytopenia following neurosurgery with a citrate tube platelet count of $77 \times 10^{3} / \mathrm{uL}$, decreased from $263 \times 10^{3} / \mathrm{uL}$ on admission.

On postoperative day three, the patient endorsed new onset leg discomfort. Lower extremity venous duplex ultrasound on postoperative day four, revealed occlusive thrombosis of the bilateral common femoral, superficial femoral, popliteal and great saphenous veins. The patient was placed on an unfractionated heparin drip after risks of VTE was determined to be greater than ICH. On postoperative day seven, the patient was switched to low molecular weight heparin (LMWH), and interventional radiology attempted to retrieve the IVC filter but was unsuccessful due to extensive thrombosis of the abdominal and pelvic vessels. Subsequent CT venogram revealed nonocclusive thrombosis of the juxtarenal IVC, above the level of the IVC with extension distally to the bifurcation of the iliac veins and bilateral common iliac, internal and external iliacs, and common femoral veins (Figure 2). Suction thrombectomy was performed on postoperative day nine to mitigate risk of pulmonary embolism. Of note, during this admission the patient's blood glucose levels were difficult to control, and consistently persistently greater than $250 \mathrm{mg} / \mathrm{dL}$ in the postoperative period.

The patient was discharged on postoperative day 11 on therapeutic LMWH. LMWH was chosen in place of a direct oral anticoagulation due to the risk of future neurosurgical intervention and availability of a reversal agent. He is continuing his care with neurosurgery and radiation oncology, feeling well and has 
not developed any further VTE since discharge.

\section{DISCUSSION}

Gliosarcomas are a rare subtype of glioblastomas occurring in a similar patient demographic, with unique histopathological features, and a grim prognosis. ${ }^{9,10}$ The histological and radiographic findings seen in our patient are consistent with the classical appearance of gliosarcoma as reported in literature. ${ }^{14,15}$ The temporal lobe is the most common location, commonly seen with surrounding vasogenic edema on imaging as seen in our patient. ${ }^{4}$ Immunohistological stains showed glial component that was rich in glial fibrillary acidic protein (GFAP). The mesenchymal components showed spindle cell sarcoma rich in reticulin and poor in GFAP. P53 is about 23\% mutated in gliosarcoma and in our patient p53 was not mutated. ${ }^{15}$

The development of VTE is a common complication in patients with any malignancy, but particularly high grade glioblastomas and gliosarcomas. ${ }^{12,13}$ The postoperative complications that occurred in our patient highlights the difficult management decisions that must be made while caring for these patients. Chemical VTE prophylaxis is challenging in high grade gliomas resections as current American Society of Clinical Oncology guidelines recommend pre- and post-surgery chemical VTE prophylaxis in cancer patients. ${ }^{16}$ However, preoperatively, chemical prophylaxis for neurosurgical procedures has been shown to increase the risk of intraoperative bleeding. ${ }^{17}$ Therefore, postoperatively, mechanical prophylaxis alone is often preferred by neurosurgeons because of the reduced risk of developing ICH when compared to chemical prophylaxis. An alternative approach to prevention of VTE is the placement of an IVC filter. However, recent data has shown that chemical prophylaxis combined with mechanical prophylaxis is superior to IVC filters and does not increase the risk of ICH when administered 24 hours after surgery. ${ }^{18}$ In addition, extensive thrombosis of the IVC filter can occur without chemical prophylaxis and removal of thrombi can potential put those patients at higher risk for adverse events. The risk of clot removal might outweigh the benefit of its placement. ${ }^{19}$

Risk factors for thrombosis, in addition to the underlying malignancy, must be considered. Prolonged immobilization in the postoperative period and history of VTE are strong risk factors for subsequent development of VTE. Obesity and diabetes are two comorbidities further increasing the patients risk of VTE. These conditions create a proinflammatory state, propagating a prothrombotic state through decreasing platelet inhibition, increasing oxidative stress, and increasing platelet activation. ${ }^{20}$ Unfortunately, literature on the management of patients with underlying hypercoagulable state undergoing intracranial surgery is limited. Our case report is unique due to the patient's underlying hypercoagulable further complicated by gliosarcoma, and subsequent development of extensive postoperative VTE despite 
mechanical prophylaxis. The development of such extensive clot burden within four days of discontinuing chemical VTE prophylaxis illustrates the difficulty of balancing the risk of VTE and ICH. While withholding chemical prophylaxis postoperatively is the standard, this case suggests patients with multiple underlying hypercoagulable states and risk factors including prior DVTs, idiopathic erythrocytosis or additional hematological and underlying medical comorbidities, and malignancies with high rates of VTE, may experience lower rates of morbidity with more aggressive postoperative pharmacologic anticoagulation. Postoperative pharmacologic anticoagulation should be done using an agent with an accessible reversal agent is paramount due to the risk of ICH.

\section{CONCLUSION}

The treatment and management of patients with high grade glioblastomas and gliosarcomas is challenging and can be further complicated by underlying medical comorbidities. VTE are common complications occurring in all cancer patients, particularly postoperatively, and hematological comorbidities inducing a hypercoagulable state may necessitate more aggressive VTE prophylaxis. The risk of bleeding must be weighed against the risk of VTE, and the anticoagulant chosen with the potential need for reversal in mind. Multiple hypercoagulable risk factors work synergistically, increasing risk of VTE by several fold. As such, patients with multiple hypercoagulable risk factors may benefit from more aggressive prophylaxis following intracranial mass resection than patients without other hypercoagulable state inducing comorbidities. 
Cooper Rowan M edical Journal, Vol. 3, Iss. 1 [2021], Art. 2

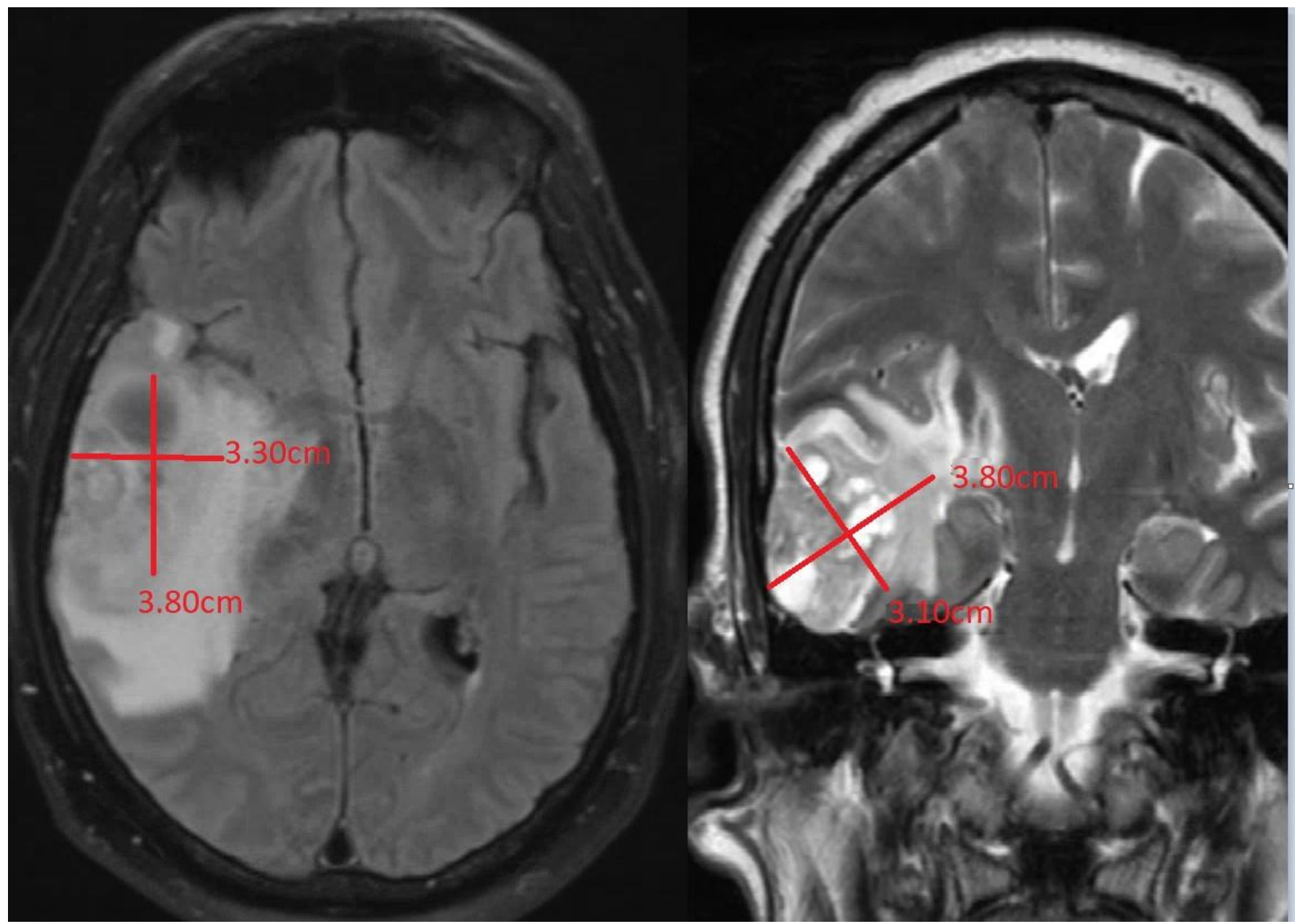

Figure 1 Right temporalgliosarcoma on magnetic resonance imaging seen in (A) Axial FLAIR and (B) T2-weighted coronal section 


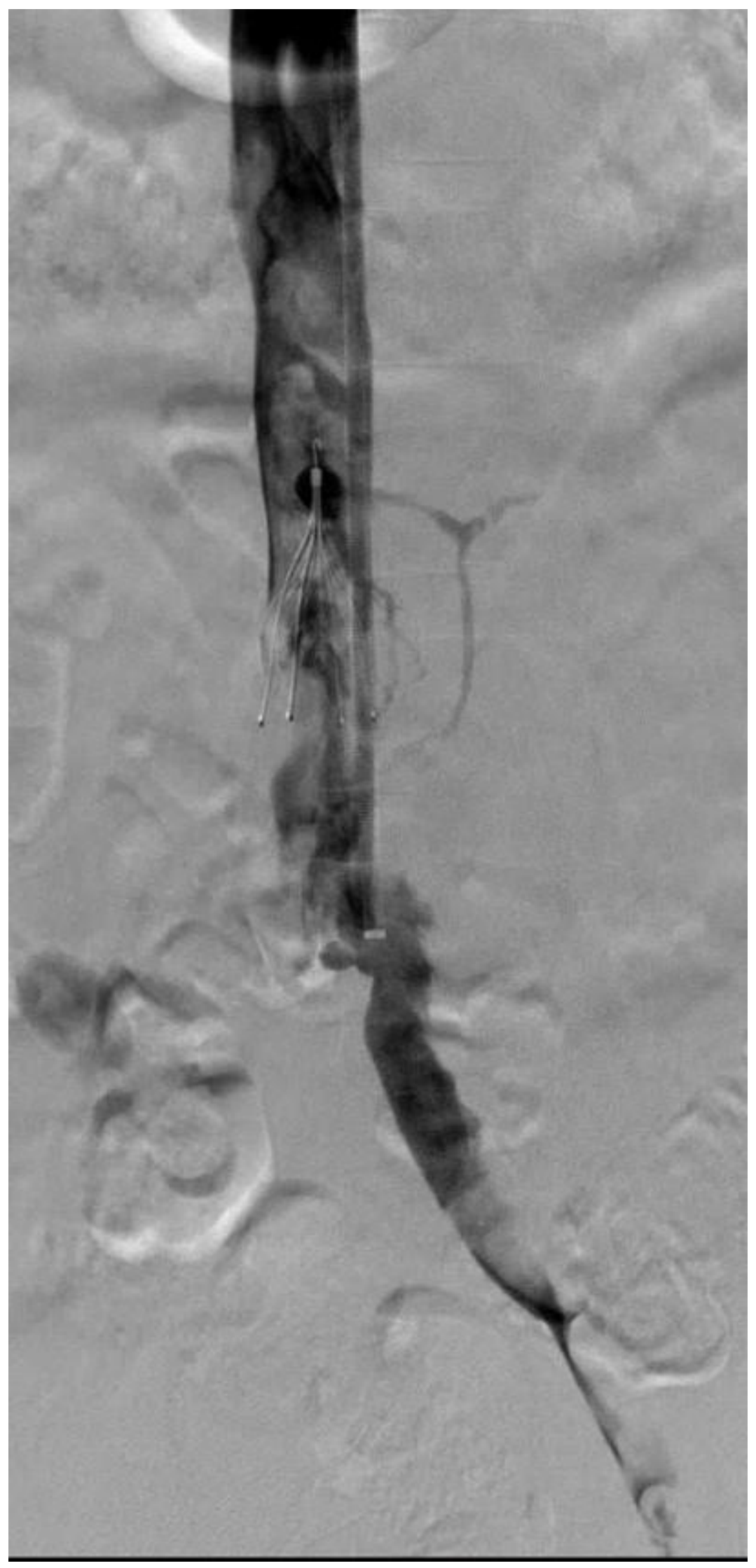

Figure 2 Fluoroscopy Showing Inferior Vena Cava Thrombosis during Attempted IVC Filter Removal 


\section{REFERENCES}

1. Louis David N., Ohgaki Hiroko, Wiestler Otmar D., et al. The 2007 WHO Classification of Tumours of the Central Nervous System. Acta Neuropathologica. 2007;114(2):97-109.

2. Burgenske D M, Yang J, Decker P A. Molecular profiling of long-term IDH-wildtype glioblastoma survivors. Neuro-oncology. 2019;21(11):1458-1469.

3. Crespo I, Vital A L, Nieto A B. Detailed Characterization of Alterations of Chromosomes 7, 9, and 10 in Glioblastomas as Assessed by Single-Nucleotide Polymorphism Arrays. J Mol Diagn. 2011;13(6):634-647.

4. Liu J, Wang L. Primary and secondary gliosarcomas: A review of clinical features and pathological diagnosis. JCO. 2018;36(15_suppl).

5. deCarvalho Ana C., Nelson Kevin, Lemke Nancy, et al. Gliosarcoma Stem Cells Undergo Glial and Mesenchymal Differentiation In Vivo. Stem Cells. 2009;28(2):N/A-N/A.

6. Barresi Valeria, Cerasoli Serenella, Morigi Federico, Cremonini Anna Maria, Volpini Mirco, Tuccari Giovanni. Gliosarcoma With Features of Osteoblastic Osteosarcoma: A Review. Archives of Pathology \& Laboratory Medicine. 2006;130(8):1208-1211.

7. Morantz Robert A., Feigin Irwin, Ransohoff Joseph. Clinical and pathological study of 24 cases of gliosarcoma. Journal of Neurosurgery. 1976;45(4):398-408.

8. McAleer Mary Frances, Brown Paul D. Therapeutic management of gliosarcoma in the temozolomide era. CNS Oncology. 2015;4(3):171-178.

9. Galanis Evanthia, Buckner Jan C., Dinapoli Robert P., et al. Clinical outcome of gliosarcoma compared with glioblastoma multiforme: North Central Cancer Treatment Group results. Journalof Neurosurgery. 1998;89(3):425-430.

10. Frandsen Simone, Broholm Helle, Larsen Vibeke Andrée, et al. Clinical Characteristics of Gliosarcoma and Outcomes From Standardized Treatment Relative to Conventional Glioblastoma. Frontiers in Oncology. 2019;9:1425-1425.

11. JENKINS E. O., SCHIFF D., MACKMAN N., KEY N. S.. Venous thromboembolism in malignant gliomas. Journal of Thrombosis and Haemostasis. 2010;8(2):221-227. 
12. Razak Norbaini Abdol, Jones Gabrielle, Bhandari Mayank, Berndt Michael, Metharom Pat. Cancer-Associated Thrombosis: An Overview of Mechanisms, Risk Factors, and Treatment. Cancers. 2018;10(10):380-380.

13. Lyman Gary H., Bohlke Kari, Khorana Alok A., et al. Venous Thromboembolism Prophylaxis and Treatment in Patients With Cancer: American Society of Clinical Oncology Clinical Practice Guideline Update 2014. Journal of Clinical Oncology. 2015;33(6):654-656.

14. Wang C, Niu X, Ren Y,Lan Z, Zhang Y.Risk Factors for Postoperative Intracranial Hemorrhage After Resection of Intracranial Tumor in 2259 Consecutive Patients. World Neurosurg. 2019;129:663-668.

15. Smith D R, Wu C C, Saadatmand H J. Clinical and molecular characteristics of gliosarcoma and modern prognostic significance relative to conventional glioblastoma. J Neurooncol. 2018;137(2):303-311.

16. Lyman Gary H, Bohlke Kari, Khorana Alok A, et al. Venous thromboembolism prophylaxis and treatment in patients with cancer: American Society of Clinical Oncology clinical practice guideline update 2014. Journal of Clinical Oncology. 2015;33(6):654.

17. Dickinson Lawrence D., Miller Lisa D., Patel Chirag P., Gupta Sanjay K.. Enoxaparin Increases the Incidence of Postoperative Intracranial Hemorrhage when Initiated Preoperatively for Deep Venous Thrombosis Prophylaxis in Patients with Brain Tumors. Neurosurgery. 1998;43(5):1074-1079.

18. Zwicker J. I., Leaf R. Karp, Carrier M.. A meta-analysis of intracranial hemorrhage in patients with brain tumors receiving therapeutic anticoagulation. Journal of Thrombosis and Haemostasis. 2016;14(9):1736-1740.

19. Levin J. M., Schiff D., Loeffler J. S., Fine H. A., Black P. McL., Wen P. Y.. Complications of therapy for venous thromboembolic disease in patients with brain tumors. Neurology. 1993;43(6):1111-1111.

20. Juhan-Vague Irène, Alessi Marie-Christine. Metabolic syndrome, haemostasis and thrombosis. Thrombosis and Haemostasis. 2008;99(06):995-1000. 